\title{
ARTÍCULOS
}

\section{DESAFÍOS DE LA EDICIÓN DE REVISTAS CIENTÍFICAS EN ACCESO ABIERTO}

\section{Challenges of publishing open access journals}

\section{Melba G. Claudio-González y Anna Villarroya}

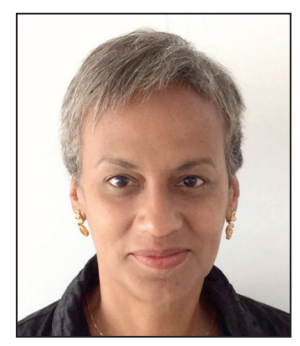

Melba G. Claudio-González es miembro del grupo de investigación a cargo del proyecto El acceso abierto a la ciencia en España del Plan Nacional del Ministerio de Ciencia e Innovación en la Universitat de Barcelona (UB), donde enmarca su tesis doctoral sobre Modelos de negocio de revistas científicas en España. Es licenciada en administración de empresas (Universidad de Puerto Rico), máster en políticas culturales $(U B)$ y especialista en modelos de gestión de redes profesionales internacionales. Co-edita la revista /encatcScholar, proyecto que diseñó para la European Network on Cultural Management and Cultural Policy con el apoyo de la Comisión Europea.

http://orcid.org/0000-0002-5788-9627

melbaclaudio@gmail.com

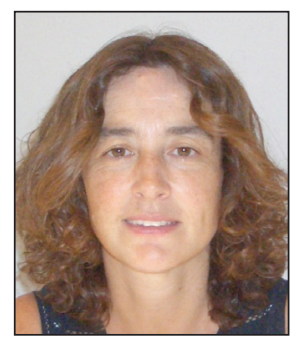

Anna Villarroya es doctora en economía del sector público y licenciada en derecho y en economía por la Universitat de Barcelona (UB). Desde 1993 es profesora del Departamento de Economía Pública, Economía Política y Economía Española de la UB, donde imparte las asignaturas Marco económico y estructura de los sectores culturales; Información y sociedad; y Economía aplicada a los medios. Es autora de varios artículos y capítulos de libro sobre economía y política cultural. http://orcid.org/0000-0002-8575-5933

annavillarroya@ub.edu

Universitat de Barcelona. Facultat de Biblioteconomia i Documentació Melcior de Palau, 140. 08034 Barcelona, España

\section{Resumen}

Una encuesta realizada a 1.280 editores de revistas científicas españolas, que tuvo un $43 \%$ ( $n=561$ ) de respuestas, ha permitido conocer sus experiencias con el acceso abierto. El análisis cuantitativo de los datos mostró que el 72\% ( $n=404)$ de los encuestados tenía experiencia con el acceso abierto o estaba inmerso en el proceso de conversión. De éstos, alrededor del $80 \%$ valoró su experiencia en términos positivos o muy positivos. Entre las dificultades más frecuentes ligadas a la adopción del acceso abierto en sus revistas destacan las limitaciones financieras y las relacionadas con la estructura organizativa.

\section{Palabras clave}

Acceso abierto; OA; Editoriales; Editores; Revistas científicas; España; Encuestas; Análisis cualitativo.

\section{Abstract}

A survey of 1,280 publishers of Spanish scientific journals ( $43 \%$ response rate; $n=561$ ) revealed their experiences with open access in scientific production. Quantitative analysis of the data showed that $72 \%(n=404)$ of respondents had experience with open access or were engaged in the conversion process. Of these, about $80 \%$ valued their experience in positive or very positive terms. Among the most common difficulties associated with the adoption of open access in their journals were the financial constraints and challenges related to organizational structure.

\section{Keywords}

Open access; OA; Scholarly journals; Publishers; Spain; Surveys; Qualitative analysis.

Claudio-González, Melba G.; Villarroya, Anna (2015). "Desafíos de la edición de revistas científicas en acceso abierto". El profesional de la información, v. 24, n. 5, pp. 517-525. 


\section{Introducción}

Durante aproximadamente dos décadas hemos sido testigos del incremento de la adopción del acceso abierto a la producción científica en España, a pesar de las dificultades y limitaciones a las que se enfrentan los actores involucrados en dicho proceso. Según Casal-Reyes et al. (2013), el mayor crecimiento en la puesta en marcha de políticas de acceso abierto se registró entre 2008 y 2012. Un análisis de la situación española de las revistas editadas en acceso abierto las sitúa en un $26 \%$ del total. Este porcentaje, que supera el $12 \%$ internacional, se puede explicar según Abadal (2012) por el distinto origen de las editoriales. Así, a diferencia de lo que ocurre en mercados líderes como Estados Unidos, Reino Unido, Países Bajos o Alemania, el panorama de la edición científica en España se caracteriza por una menor presencia de editoriales comerciales. El hecho de que aproximadamente tres cuartas partes sean editoriales sin ánimo de lucro (dependientes de universidades, centros públicos de investigación, sociedades científicas y asociaciones y colegios profesionales) las hace mucho más proclives a la filosofía del movimiento del acceso abierto. De ahí que comparativamente las editoriales españolas hayan avanzado con mayor rapidez hacia este modelo.

En este marco, y a pesar del consenso en torno a las bondades del acceso abierto a la producción científica, centradas principalmente en la importancia de la difusión del conocimiento y en su compromiso en contra de los monopolios editoriales (García-Arístegui; Rendueles, 2014), el debate sobre las limitaciones y dificultades que pueden frenar su desarrollo sigue vigente. Si bien hay instituciones de gran prestigio asentadas en la comunidad científica que defienden los formatos abiertos, la confrontación entre conocimiento cerrado y abierto sigue existiendo (Alonso-Puelles; Echevarría-Ezponza, 2014). Con dicho debate como telón de fondo, este trabajo explora la valoración general y actitudes de los editores en torno a su experiencia con la edición de revistas científicas en acceso abierto.

\section{Método de investigación}

Los resultados de este trabajo proceden de una encuesta auto administrada vía Limesurvey entre 1.280 editores de revistas científicas españolas incluidas en la base de datos Dulcinea, que recoge los derechos de copyright y las condiciones de autoarchivo de las revistas españolas (Melero et al., 2009). http://www.limesurvey.org http://www.accesoabierto.net/dulcinea deaba la opinión de los editores en torno a las oportunidades y amenazas para la edición de revistas científicas en España, su valoración sobre la experiencia con el acceso abierto, así como las posibles dificultades de adoptar dicha filosofía. También se ofreció un espacio para anotaciones abiertas donde pudieran argumentar la valoración de su experiencia y especificar dificultades no listadas en la pregunta cerrada.

A pesar del consenso sobre las bondades del acceso abierto, el debate sobre las limitaciones y dificultades que afectan su desarrollo sigue vigente

Con los resultados obtenidos se realizó el análisis estadístico de los datos y un análisis cualitativo de las anotaciones abiertas con apoyo del programa Atlas.ti, que permitió obtener lógicas de interpretación mediante el tratamiento sistemático de la información. Asimismo, facilitó el proceso de codificación de los conceptos centrales de las anotaciones en función de si éstas fueron expresadas en un sentido positivo, negativo o neutral. El uso de estos programas informáticos apoya el proceso de análisis a través de la codificación, el proceso de redacción de reflexiones analíticas, el enlazado (o vinculación) y las funciones de visualización de la red. El análisis del contenido latente implica una primera fase de organización, procesamiento y análisis de los datos que permite trabajar, con posterioridad, en la abstracción e interpretación de los mismos. Si bien estos procesos cualitativos se caracterizan por seguir una secuencia no lineal (GarcíaHernández; Manzano-Caudillo, 2010), el análisis del contenido de las anotaciones se llevó a cabo siguiendo una ruta más o menos secuencial para la identificación, codificación y categorización subyacente en los datos (figura 1).

Las editoriales que respondieron la encuesta presentan las siguientes características:

- existe un claro predominio de las de titularidad universitaria (37\%), a la que siguen las asociaciones y sociedades científicas (27\%) y los organismos de investigación (16\%); - a nivel geográfico, Madrid (27\%), Cataluña (18\%) y Andalucía (13\%) son las comunidades autónomas con mayor representatividad;

- por áreas temáticas, un $68 \%$ pertenecen a humanidades y ciencias sociales, divididas a partes iguales en artes y humanidades (34\%) y ciencias sociales y jurídicas (34\%);

La encuesta, que se mantuvo abierta entre los meses de noviembre de 2012 y marzo de 2013, obtuvo una tasa de respuesta del $43 \%$ ( $n=561)$. Para ello se siguió el Tailored design method (Dillman; Smyth; Christian, 2009), que se ha convertido en una referencia estándar en la investigación por encuestas. El cuestionario incluyó tres preguntas en las que se son-

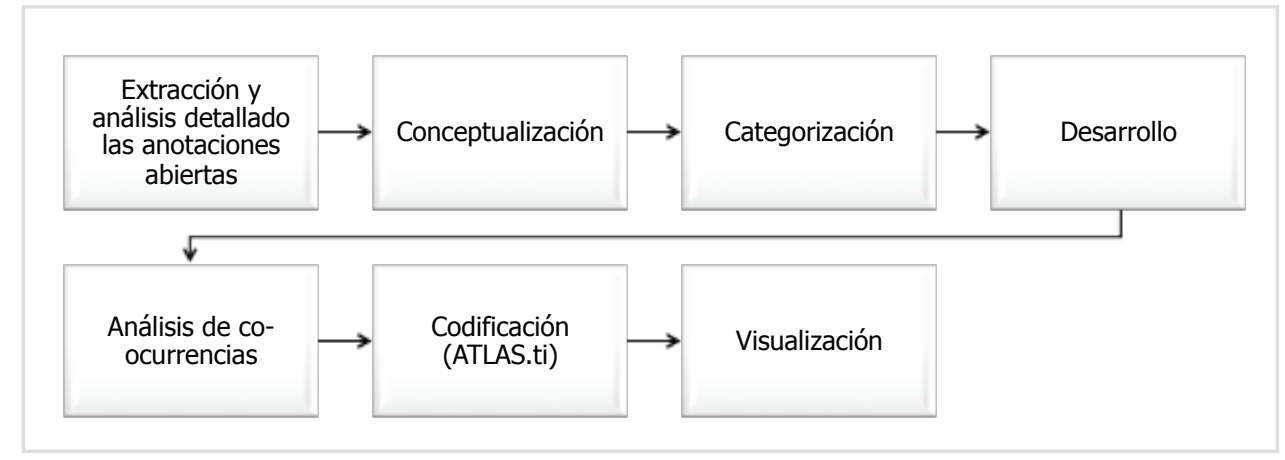

Figura 1. Fases de elaboración del análisis cualitativo sobre la experiencia de los editores con el acceso abierto 
- un $50 \%$ publica tanto en formato digital como impreso, el 33\%, sólo en formato digital, y un $20 \%(n=111)$, en formato impreso;

- en cuanto al idioma, el $42 \%$ utiliza el castellano y el inglés en proporciones variables y el $34 \%$ combina el castellano con las lenguas cooficiales u otros idiomas.

\section{Valoración general}

En un escenario en el que la mayoría del material publicado en las revistas científicas se ha financiado con dinero público (European Commission, 2012) y donde los recortes en los presupuestos gubernamentales destinados a apoyar la producción científica han sido alarmantes, no es de extrañar que las restricciones económicas se vean ampliamente comentadas en la bibliografía. Sin embargo, y probablemente como consecuencia de ello, la dimensión estratégica del modelo de negocio de las revistas científicas ha cobrado especial relevancia. Pocos dudarían en reconocer que la dimensión estratégica es un factor clave en el contexto de cambios que vive el sector, en el que la capacidad de transformación y adaptación de la actividad editorial al mercado actual se torna una necesidad urgente. Dicho proceso no está exento de dificultades que potencialmente frenan el desarrollo de la cultura del acceso abierto a la producción científica. Y es en este contexto que consideramos importante sondear la opinión de los editores españoles sobre el balance de su experiencia.

De los 561 editores de revistas científicas españolas que respondieron la encuesta, más del $70 \%$ declaró tener experiencia con el acceso abierto o estar inmerso en el proceso de conversión (gráfico 1). Poco más del $20 \%$ declaró no tener experiencia, del cual aproximadamente la mitad respondió que ni siquiera se lo había planteado.

En línea con estudios previos, como el de Abadal (2014), el análisis por áreas de conocimiento pone de relieve una mayor expansión del acceso abierto en las áreas de ingeniería y arquitectura, multidisciplinares y ciencias sociales y jurídicas. En estas áreas el tener experiencia con el acceso abierto o el encontrarse en proceso de conversión alcanza porcentajes próximos al 80\%, 10 puntos por encima de la media.

De todas las revistas con experiencia en la edición en acceso abierto $(n=333)$, un $96,4 \%(n=321)$ ofreció su valoración sobre la misma. Aproximadamente el $80 \%$ de éstas, además de valorar la experiencia, añadieron una breve anotación explicando el área de gestión o el resultado específico sobre el cual sustentaban su opinión.

Tras el proceso de codificación, las categorías resultantes fueron las siguientes:

- Experiencia muy positiva: afirmaciones que incluyeron los términos "muy positivo", "altamente recomendable", "excelente", "formidable" o "muy bueno".
- Experiencia positiva: recoge las afirmaciones que describieron la experiencia con adjetivos como "positiva" o "buena", las que describieron positivamente uno o más resultados de la experiencia aunque no usen dichas palabras, y las que expresaron que el acceso abierto es una parte irrenunciable de su filosofía operativa y estratégica.

- Con reservas: afirmaciones que partieron de una valoración positiva, pero añadieron algún argumento que limitaba dicha afirmación en algún aspecto. Por ejemplo, "positivo, pero menor de lo esperado" o "positiva, pero económicamente penosa".

- Neutra o difusa: afirmaciones que, sin expresar una valoración negativa, tampoco enunciaron argumentos que reflejaran de forma clara un sentido positivo de la valoración. Por ejemplo, "apenas hay diferencia". También se recogen en esta categoría los comentarios que, por razones variadas, señalaron que no desean o no pueden ofrecer una valoración. Por ejemplo, "es pronto para saberlo"; "no tenemos indicadores para valorar la experiencia" o "la experiencia no es valorable".

- Negativa: afirmaciones que describieron la experiencia con adjetivos como "negativa" o "mala", o que puntualizaron negativamente uno o más resultados de la misma, aunque no usaran dichas palabras.

Tras el análisis cualitativo de los datos, alrededor del $80 \%$ valoró su experiencia en términos positivos o muy positivos (gráfico 2).

El análisis por áreas de conocimiento de las revistas y tipo de entidad propietaria no reveló diferencias importantes en la valoración de la experiencia. Por áreas de conocimiento, la única excepción se aprecia en las revistas de ciencias y de ciencias de la salud, con una mayor presencia de argu- 


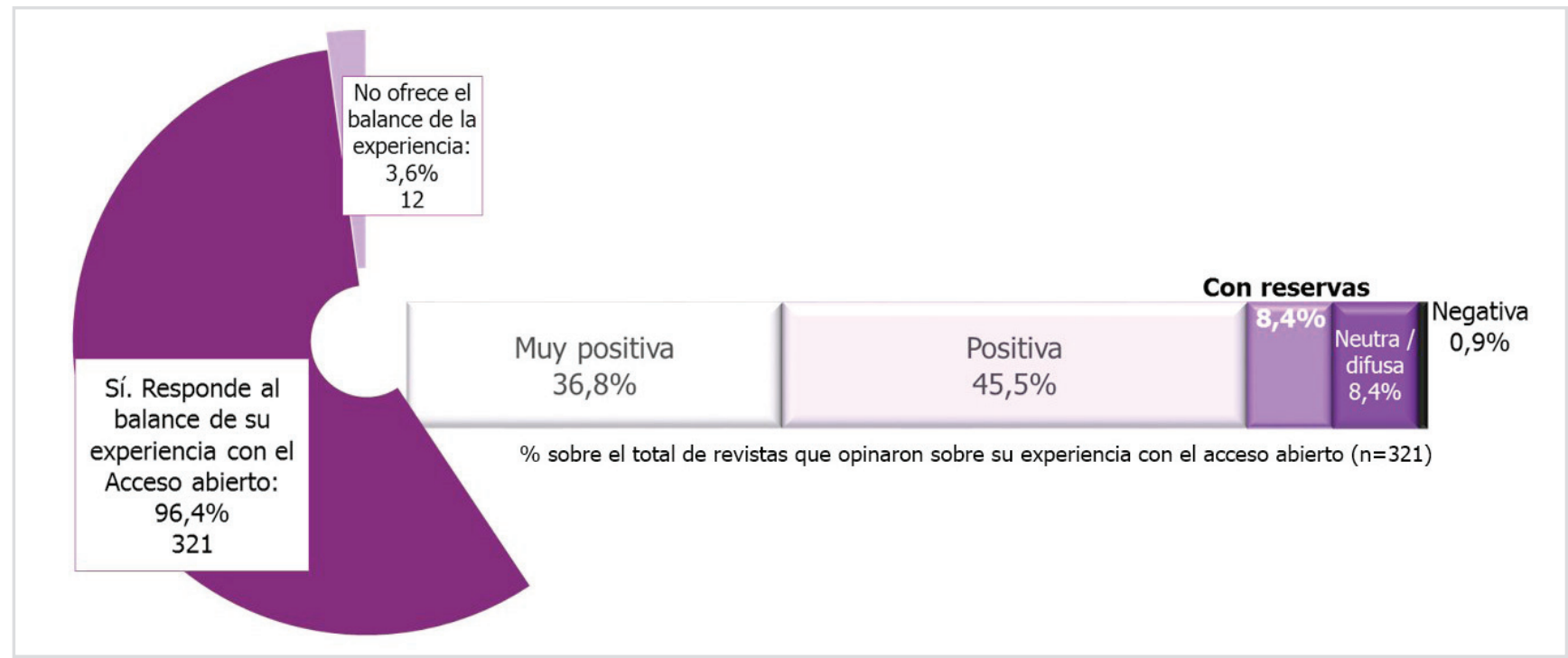

Gráfico 2. Balance de la experiencia con la edición en acceso abierto.

$\%$ sobre el total de revistas con experiencia con el acceso abierto $(n=321)$

mentos con reservas (14\%-16\% respectivamente) versus un 6-8\% del resto de áreas. Por su parte, el análisis por tipo de entidad propietaria sólo reveló un mayor predominio de las valoraciones muy positiva y positiva de las editoriales universitarias y de las pertenecientes a organismos públicos de investigación (un $87 \%$ frente al promedio del $82 \%$ ).

\section{Orientación de los argumentos}

Con el objetivo de facilitar el análisis de los datos, que se muestra en los próximos apartados, las cinco categorías de clasificación anteriores se redujeron a tres:

- valoraciones favorables al acceso abierto;

- desfavorables;

- neutrales.

Una amplia mayoría de las anotaciones (93\%) aportaron argumentos favorables, mientras que sólo un $11 \%$ señaló argumentos desfavorables. Un dato curioso de este último resultado es que, si bien los argumentos positivos los aportaron mayoritariamente las personas cuyo balance de la experiencia también fue positivo, casi un $70 \%$ de los argumentos desfavorables los aportaron encuestados cuyo balance de la experiencia partía de una valoración positiva, pero con reservas (gráfico 3).

\section{Argumentos favorables}

Es fácil encontrar en la bibliografía referencias a los aspectos positivos del acceso abierto, empezando por los beneficios globales asociados a la eliminación de las barreras de acceso. Algunos estudios abordan el incuestionable compromiso político social que subyace tras esta ideología, y otros exploran sectores o ámbitos muy específicos para reforzar determinadas ventajas.

En el primer grupo se encuentran trabajos como el de Solaro y Lamberti (2011), que identifican cuatro ventajas:

- elimina la necesidad de grandes presupuestos para la adquisición del acceso;

- favorece la difusión científica;
- facilita la tarea editorial;

- colabora en la democratización del conocimiento.

También están aquellos que defienden la creencia generalizada de que el intercambio de datos facilitará un progreso científico más rápido y una reducción de la duplicidad innecesaria de esfuerzos científicos (Parker, 2013). Parker destaca otros tres argumentos que impulsan el desarrollo del acceso abierto:

- las obligaciones de reciprocidad en las que los resultados de la investigación financiada por el sector público deben estar libremente a disposición de los que han pagado por ello;

- los modelos basados en la suscripción de la edición son injustos y explotadores;

- la publicación en acceso abierto ofrece la posibilidad de alejarse de un enfoque impulsado por objetivos de marketing y puntos de vista excesivamente rígidos acerca de los límites disciplinarios y de los formatos de publicación.

\section{Entre los aspectos positivos del acceso abierto están la visibilidad, el favorecer el acceso a la ciencia, y el aumento de lectores, visitas y descargas}

Otra postura esencial en este primer grupo es la de la Comisión Europea que en repetidas ocasiones ha alentado a los estados miembros a poner los resultados de las investigaciones financiadas con fondos públicos en la esfera pública a fin de fortalecer la ciencia y la economía basada en el conocimiento.

En el segundo grupo de estudios que abordan aspectos más específicos sobre los efectos de la adopción del acceso abierto se encuentran trabajos como el de Riera y Aibar (2013) que, desde el ámbito de la medicina intensiva, analizan si el acceso abierto favorece el impacto de los artículos científicos en términos de citación. Estos autores concluyen que 
la publicación en abierto [y el Índice de Hirsch del primer autor] favorecen el impacto de los artículos científicos. Otro ejemplo es el trabajo de Björk y Solomon (2012) destinado a dar respuesta al debate sobre si la proliferación del acceso abierto dañaría el sistema de revisión por pares y pondría en riesgo la calidad de la publicación de revistas científicas. Para ello compararon el impacto científico de revistas de suscripción con el de revistas en abierto. Sus resultados indicaron que las revistas en acceso abierto indexadas en Web of Science o Scopus están alcanzando el mismo impacto científico y de calidad que las de suscripción, particularmente las de biomedicina y las financiadas por cargos de procesamiento por artículo (APCs).

En este marco orientador proporcionado por la bibliografía, el análisis de las anotaciones positivas recogidas en el sondeo permitió identificar nueve categorías:

1. Mejora la visibilidad, vinculada también a la internacionalización de las revistas.

2. Favorece y agiliza el acceso a la ciencia, y aumenta las citas.

3. Ayuda al aumento de lectores, visitas y descargas.

4. Es muy importante y socialmente imprescindible.

5. Es sencillo, mejora la gestión y permite innovar y salir de modelos obsoletos.

6. Los costes son más bajos.

7. Aumentan las contribuciones y permite una selección más consistente.

8. Favorece la calidad de contenidos.

9. Es fundamental para las revistas pequeñas.

Las tres primeras fueron las más repetidas entre las anotaciones.

El quinto argumento, que relaciona la sencillez del proceso con los aspectos favorables del acceso abierto, contrasta con el planteamiento de Dogra (2015), quien identifica como un mito la idea de que "publicar en una revista de acceso abierto es fácil".

\section{Argumentos desfavorables}

A pesar del discurso dominante en torno a la idea de que el acceso abierto conduce a un aumento en el número de citas y acelera el progreso de la investigación, la productividad y la difusión del conocimiento, algunos autores señalan la existencia de cuestiones todavía sin resolver que apuntan a que es tan imprescindible como discutible (Dai et al., 2014). Entre otras, se han planteado cuestiones como la exhausti- vidad del proceso de revisión por pares, el rigor académico o el obstáculo de los cargos por procesamiento de artículos (Das, 2015). Barreiro (2013), por ejemplo, plantea el problema de las revistas en acceso abierto creadas por grupos de científicos aislados y no amparadas por sociedades científicas. En éstas, según la autora, todavía existen reservas acerca de la calidad del proceso de revisión por pares.

Entre los argumentos desfavorables a la experiencia con el acceso abierto se encontró que tiene una financiación débil o resulta poco viable

Varias de estas preocupaciones apuntadas en la bibliografía académica salieron a relucir entre las anotaciones de los editores encuestados. El resultado de la categorización de los argumentos desfavorables a la experiencia con el acceso abierto quedó recogido en ocho categorías lideradas cuantitativamente por la opinión de que el acceso abierto tiene una financiación débil o resulta poco viable:

1. Financiación débil o poco viable.

2. Percepción negativa de la gratuidad y el acceso libre.

3. Falta de apoyo técnico y legal.

4. Es un proceso complejo.

5. El embargo empobrece.

6. Fuerte competencia y control de las empresas editoriales.

7. Desarrollo limitado del factor de impacto.

8. Carencia de políticas que apoyen la publicación en español.

\section{Principales limitaciones}

También se exploró si los encuestados percibían aspectos que podrían limitar la adopción del acceso abierto en sus revistas. Cabe destacar que en esta pregunta podían opinar tanto los editores con experiencia con el acceso abierto, 
como los que estaban en proceso de conversión y los que aún no se lo habían planteado. En esta pregunta se obtuvo una tasa de respuesta del $86,3 \%(n=484)$.

Las limitaciones financieras fueron las marcadas mayoritariamente $(45 \%)$ por los editores. A éstas les siguieron las limitaciones organizativas (31\%) y las tecnológicas (24\%). Solamente un $1 \%$ de los encuestados no identificó dificultad alguna (gráfico 4).

Las anotaciones abiertas en las que los encuestados pudieron argumentar su respuesta o expresar otras dificultades específicas, fueron estudiadas en profundidad en el análisis cualitativo, obteniendo como resultado, seis grandes categorías:

\subsection{Conservadurismo}

Recoge dificultades que tienen que ver con la resistencia al cambio por parte de cualquiera de los actores involucrados, ya sean los editores, autores o instituciones financiadoras; por el área científica en la que se circunscribe la revista o por el usuario que valora tener un producto final material.

Los editores señalaron además tres formas específicas en las que se manifiesta dicha resistencia:

- peso de la tradición, entendido como el conservadurismo de los agentes que están implicados en la toma de decisiones y en los asuntos relacionados con la manera de gestionar la revista;

- peso de los hábitos, no sólo de los editores, sino también de algunos bibliotecarios y autores reticentes a cambiar los hábitos adquiridos bajo las políticas editoriales tradicionales;

- peso de la inercia, que recoge a quienes argumentan que no optar por el cambio es simplemente una cuestión de preferencia por la edición impresa. Se basan pues en la percepción de que el entorno digital es efímero o que sienten cierto fetichismo por el papel. Sin embargo, ante un cambio ineludible hay quien reconoce que podría tener interés por el acceso abierto, siempre y cuando estuviera sujeto a un embargo de un año.

\subsection{Cultura del acceso abierto no consolidada}

En este punto los encuestados identificaron al menos cuatro áreas de mejora:

\section{Necesidad de ampliar y clarificar las nociones sobre el ac- ceso abierto}

Se identificaron importantes carencias en ese sentido y, en concreto, ciertas dudas sobre el concepto de propiedad en el contexto de la publicación científica. Este resultado coincide con el estudio de Casal-Reyes et al. (2013), quienes llegaron a la conclusión de que si bien la mitad de las instituciones universitarias en España disponen de servicios de ayuda a los editores, los investigadores requieren un mayor asesoramiento sobre cuestiones relacionadas con la edición y, más concretamente, con la edición en acceso abierto.

\section{Mayor compromiso social y político}

Los editores pusieron de relieve cómo el acceso abierto en sí mismo no supone grandes dificultades, pero puede parecerlo en la medida en que se carezca de un compromiso social y político, especialmente en el ámbito de la producción científica financiada con dinero público. Asimismo destacaron que la falta de compromiso social y político alimenta el miedo a no tener herramientas adecuadas para moverse en el marco legal del entorno digital y de las políticas de difusión científica de las instituciones públicas.

\section{Autonomía de gestión}

Estas anotaciones hicieron referencia a la fuerte dependencia respecto a la editorial comercial que distribuye sus revistas, y participa en la toma de decisiones del proyecto editorial en general.

\section{Estructura organizativa}

Algunos encuestados señalaron como asignaturas todavía pendientes en el camino hacia la consolidación de la cultura del acceso abierto en España la necesaria atención a las fisuras de la estructura organizativa y la profesionalización de la edición de revistas científicas.

\subsection{Percepción de pérdida}

Otras dificultades derivadas de la adopción de la filosofía de acceso abierto fueron expresadas a partir de una percepción de pérdida que podía manifestarse de una forma u otra no sólo entre editores, sino también entre los autores y los lectores. En este sentido, los dos inconvenientes del acceso abierto puestos de relieve por Solaro y Lamberti (2011), recogidos también en los resultados de este estudio, son las reticencias a la disminución de la calidad científica, y a que los índices de impacto de estas revistas aún no son muy elevados.

Los encuestados apuntaron también que, si bien se perciben cambios en ese sentido, aún no está superada la idea de que una revista electrónica tiene menos prestigio que la revista impresa. A esto se añade la tendencia a desvalorizar las revistas por cuestiones formales y a asociar el acceso abierto con revistas de menor impacto científico. De
Gráfico 4. Principales dificultades para adoptar la filosofía de acceso abierto. $\%$ sobre el total de encuestados ( $n=561)$. Tasa de respuesta: $86,3 \%(n=484)$ 
ahí que algunos editores apunten a que prefieren optar por medidas intermedias manteniendo la versión en papel y abriendo el acceso después de un período de embargo.

Por otro lado hay comentarios que señalan que algunos autores son renuentes a publicar en abierto por el supuesto escaso control de calidad que se asocia a este entorno y por la percepción de pérdida de derechos de propiedad intelectual. También desde la perspectiva de los autores, Abadal (2012) ha llamado la atención sobre la disparidad entre su valoración sobre el acceso abierto y su actuación como autores. En este sentido, a pesar de que el $90 \%$ de los científicos considera muy positivo disponer de artículos en acceso abierto, la realidad es que sólo el $20 \%$ de éstos están disponibles en esa modalidad.

Por último, la anotación más radical en este grupo es la que hace mención a la falta de equidad a la hora de valorar las revistas, apuntando a que existe una diferente vara de medir por parte de las grandes organizaciones que controlan las bases de datos y las citas, ya que éstas siguen vinculando la calidad de contenidos a la revista impresa.

\subsection{Otras carencias}

Algunos encuestados apuntaron otras posibles carencias, que si bien inciden en la falta de información fiable y precisa sobre qué es el acceso abierto, añaden otros matices, como:

- falta de entusiasmo y de confianza en las bondades del conocimiento compartido:

- falta de visión estratégica;

- nociones equívocas con respecto a la dinámica de gestión de la información desde el entorno digital. Por ejemplo, se hace una referencia específica a quienes tienen la idea de que se puede cobrar por las visitas;

- falta de paciencia y de entusiasmo por el trabajo que se está realizando.

Esta última observación no es menor si tenemos en cuenta que en un contexto de cambio de paradigma, la capacidad para afrontar y adaptar el proyecto editorial con sosiego y buen ánimo puede ser vital para la obtención de unos buenos resultados.

\subsection{Conflicto de intereses}

Una parte de los encuestados hicieron hincapié en las incompatibilidades que son capaces de frenar el cambio hacia el acceso abierto, en tanto que seguir adelante conllevaría modificar o romper acuerdos con terceros. Entre las incompatibilidades más destacadas se encuentran:

- revistas que utilizan la versión impresa para gestionar importantes intercambios que no serían compatibles con una publicación digital abierta;

- revistas editadas desde sociedades científicas que se financian con el pago de cuotas de sus socios. Ofrecer acceso abierto a la revista supondría para los socios la pérdida de un privilegio intrínseco al pago de la cuota, y para los editores, el riesgo de perder los ingresos de los que dependen;

- conflictos de intereses económicos vistos más bien desde un punto de vista ideológico que contable. Dicho de otro modo, las dificultades podrían surgir en el momento en el que la voluntad de hacer negocio sea lo que inspire la misión de un proyecto editorial basado en la filosofía de acceso abierto. Esta idea podría relacionarse con una de las críticas que señala Dogra (2015) y que apunta al mito de que la aceptación del manuscrito por parte de las revistas de acceso abierto está motivada por las ganancias monetarias;

- dificultad de modificar las condiciones de publicación, principalmente en el caso de las revistas que tienen preacuerdos con editoriales comerciales.

\section{Aún no está superada la idea de que una revista electrónica tiene menos prestigio que la revista impresa}

\subsection{Complejidades del proceso de búsqueda de re- cursos estables}

Esta última categoría reúne anotaciones sobre la dificultad de buscar recursos que aseguren la viabilidad del proyecto editorial, empezando por la falta de recursos humanos.

También en este grupo se hace referencia a dos dificultades añadidas:

- complejidad que supone lidiar con la periodicidad inestable de los recursos, aun teniéndolos;

- reducción de los incentivos a la suscripción.

La mención a la falta de tiempo se ha dado en un contexto que podría llevarnos a pensar que existe cierto grado de "normalización" o de "resignación" con la falta de recursos en general. En proyectos editoriales que existen gracias al trabajo ingente $y$, en muchos casos, voluntario de profesionales comprometidos con la revista, no es de extrañar que éstos hagan referencia a la necesidad de tiempo, cuando han de compatibilizar la gestión de la revista con su actividad profesional principal.

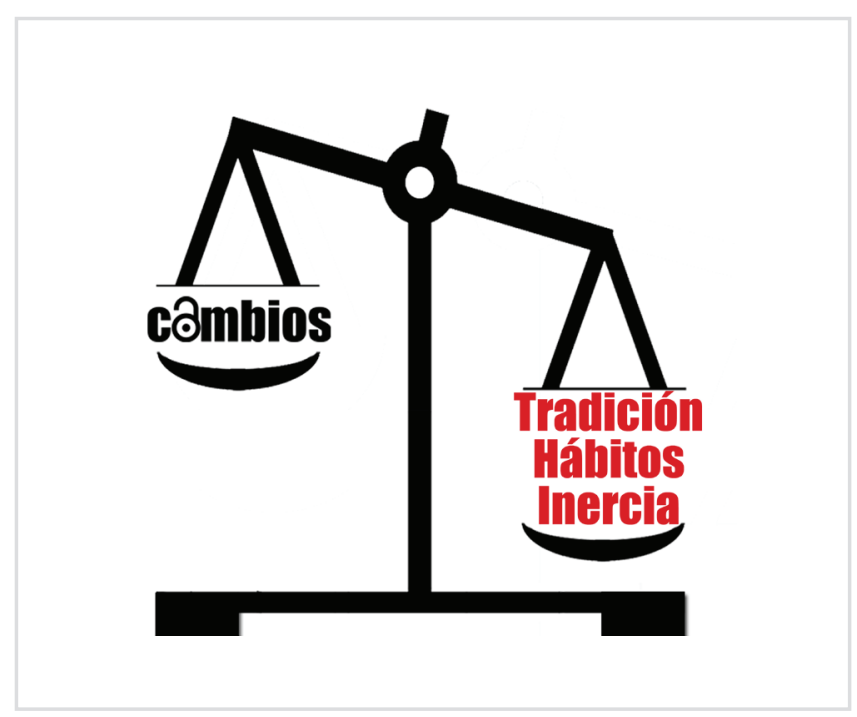




\section{Conclusiones. Pros y contras de una experiencia mayoritariamente positiva}

El estudio ha permitido ver cómo alrededor del $80 \%(n=263)$ del total de editores encuestados que dijo tener experiencia con el acceso abierto la ha valorado en términos positivos o muy positivos.

Se ha puesto de relieve cómo a pesar de que la experiencia es positiva, el porcentaje de encuestados que aportaron una visión crítica y sincera sobre las limitaciones y dificultades a las que aún se enfrentan no ha sido marginal.

En suma, "los contras" podrían resumirse en dos llamadas de atención:

- Puntos débiles: los encuestados identificaron como desfavorables al menos ocho aspectos de la gestión editorial del acceso abierto, y señalaron una serie de situaciones que podrían dificultar o frenar la adopción de esa filosofía.

- Necesidad de profundizar en lo que realmente implica consolidar la cultura del acceso abierto, atendiendo a las áreas de mejora identificadas. Es en esas áreas de mejora en las que merece la pena centrar la atención para así poder fortalecer y favorecer el desarrollo de la producción de las revistas científicas en España.

Puesto que las limitaciones financieras y las relacionadas con la estructura organizativa han sido las áreas de mejora más señaladas, otras conclusiones a tener en cuenta apuntan a la necesidad de clarificar conceptual y operativamente las implicaciones que supone la adopción de la filosofía del acceso abierto entre los editores científicos. A este ejercicio de clarificación conceptual, cabe añadir la necesidad de ampliar los conocimientos sobre las distintas áreas de gestión de los proyectos editoriales, incluyendo la aplicación de nuevos modelos de negocio (Villarroya el al., 2012). En relación con este último punto, cabe destacar la llamada a reflexionar y a someter a un proceso de renovación la visión estratégica de algunos proyectos editoriales científicos que aún se encuentren anquilosados en estructuras tradicionales y resistentes a los cambios.

Por último, hay que destacar la llamada de los editores científicos a la necesidad de reflexionar sobre el compromiso social y el reforzamiento de las políticas públicas que afectan el acceso abierto a la ciencia, prestando especial atención a la gestión del conocimiento generado a través de la investigación financiada con dinero público.

\section{Agradecimientos}

Esta investigación se ha realizado en el marco del proyecto del Plan Nacional El acceso abierto (open access) a la ciencia en España: Análisis del grado de implantación y de la sostenibilidad de un nuevo modelo de comunicación científica, financiado por el Ministerio de Ciencia e Innovación (Referencia: CS02011-29503-C02-01).

\section{Bibliografía}

Abadal, Ernest (2012). Acceso abierto a la ciencia. Barcelona: Editorial UOC, colección El profesional de la información, n. 5. ISBN: 9788497885485
Abadal, Ernest (2014). "El acceso abierto en humanidades". Humanidades digitales: una aproximación transdisciplinar. Janus, Anexo 2, pp. 17-32.

$h t t p: / / g o o . g l / E d z s 7 L$

Alonso-Puelles, Andoni; Echevarría-Ezponza, Javier (2014). "Lucha de paradigmas: leyes, ciencia y activismo en el mundo open". Argumentos de razón técnica, marzo, n. 17, pp. 21-38. http://institucional.us.es/revistas/argumentos/17/art_2.pdf

Barreiro, Esther (2013). "Open access: is the scientific quality of biomedical publications threatened?". Archivos de bronconeumología, diciembre, v. 49, n. 12, pp. 505-506. http://dx.doi.org/10.1016/j.arbr.2013.10.003

Björk, Bo-Christer; Solomon, David (2012). “Open access versus subscription journals: a comparison of scientific impact". BMC Medicine, julio, v. 10, n. 1, pp. 73. http://doi.org/10.1186/1741-7015-10-73

Casal-Reyes, Mabela; Borgoñós, María-Dolores; Casaldàliga, Anna; Gómez, Javier; Guijarro, Concha; Ortiz, Eva; Pascual-del-Pobil, Almudena; Rodríguez, Fernando; Terroba, Isabel (2013). "El acceso abierto en las universidades españolas: estado de la cuestión y propuestas de mejora". MEI: Métodos de Información. Colegio Oficial de Bibliotecarios y Documentalistas de la Comunidad Valenciana.

http://www.metodosdeinformacion.es/mei/index.php/mei/ article/view/IIMEI4-N6-055090

Dai, Ni; Xu, Dingyao; Zhong, Xiyao; Li, Li; Ling, Qibo; Bu, Zhaode (2014). "Publishing in open access era: focus on respiratory journals". Journal of thoracic disease, v. 6, n. 5, pp. 564-567.

http://doi.org/10.3978/j.issn.2072-1439.2014.03.18

Das, Anup-Kumar (2015). “Open access: History and developments". En: Unesco. Introduction to open access [Open access for library schools, 1]. Unesco, Paris, pp. 17-30. ISBN: 9789231000744

http://eprints.rclis.org/24903

Dillman, Don A.; Smyth, Jolene D.; Christian, Leah M. (2009). Internet, mail, and mixed-mode surveys: The tailored design method ( $3^{\text {rd }}$ ed.). Hoboken, NJ: John Wiley Co. ISBN: 9780471698685

Dogra, Vikram (2015). "Myths about publishing in an open access journal". Journal of clinical imaging science, n. 5, pp. 26. http://doi.org/10.4103/2156-7514.156140

European Commission (2012). Recommendations on access to and preservation of scientific information.

http://goo.gl/N9h7Hc

https://goo.gl/cE3b9n

García-Arístegui, David; Rendueles, César (2014). "Abierto, libre y público: los desafíos políticos de la ciencia abierta". Argumentos de razón técnica: Revista española de ciencia, tecnología y sociedad, y filosofía de la tecnología, n. 17, pp. 45-64. http://dialnet.unirioja.es/servlet/articulo?codigo $=4935407$

García-Hernández, Gloria E.; Manzano-Caudillo, Jesús (2010). "Procedimientos metodológicos básicos y habilidades del investigador en el contexto de la teoría fundamenta- 
da". Iztapalapa. Revista de ciencias sociales y humanidades, v. 31, n. 69, pp. 17-39.

http://dialnet.unirioja.es/servlet/articulo?codigo $=3834350$

Melero, Remedios; Abad, María-Francisca; Abadal, Ernest; Amat, Carlos B.; Giménez, Francesc; Rodríguez-Gairín, Josep-Manel; Rodríguez, Nerea (2009). "Dulcinea: Iniciativa para el análisis de los derechos de copyright y autoarchivo de revistas científicas españolas". En: XI Jornadas Españolas de Documentación. Fesabid'09, Zaragoza, 20-22 mayo.

http://www.fesabid.org/zaragoza2009/actas-fesabid-2009/117-121. $p d f$

Parker, Michael (2013). "The ethics of open access publishing". BMC medical ethics, v. 14, n. 16.

http://doi.org/10.1186/1472-6939-14-16
Riera, María; Aibar, Eduard (2013). “Does open access publishing increase the impact of scientific articles? An empirical study in the field of intensive care medicine". Medicina intensiva, v. 37, n. 4, pp. 232-240.

http://doi.org/10.1016/j.medin.2012.04.002

Solaro, Gicelt; Lamberti, Alicia (2011). Open access: un nuevo camino para recorrer en la sociedad del conocimiento. http://eprints.rclis.org/handle/10760/17265

Villarroya, Anna; Claudio-González, Melba; Abadal, Ernest; Melero, Remedios (2012). "Modelos de negocio de las editoriales de revistas científicas: implicaciones para el acceso abierto". El profesional de la información, v. 21 n. 2, pp. 129135.

http://dx.doi.org/10.3145/epi.2012.mar.02

\section{Nueva colección EPI Scholar}

\section{Libros académicos y científicos de Información y Documentación}

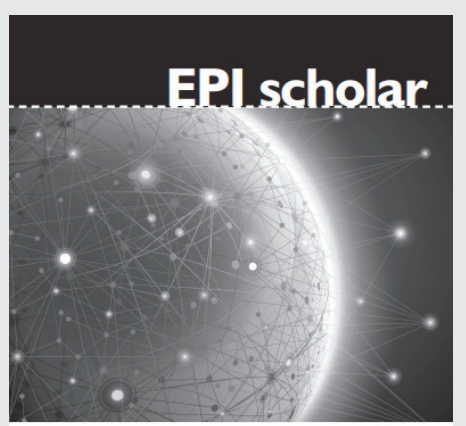

CIBERMETRÍA MIDIENDO EL ESPACIO RED ENRIQUE ORDUNA-MALEA ISIDRO F. AGUILLO

\section{Cibermetría. Midiendo el espacio red de Enrique Orduña-Malea e Isidro F. Aguillo}

A pesar del crecimiento de la disciplina de la Cibermetría desde mediados de la década de los noventa, son escasos los libros académicos o manuales dedicados en exclusiva a la misma desde un contexto de las ciencias de la información y documentación. Este libro pretende cubrir este claro hueco en la literatura tanto nacional como internacional.

Orduña-Malea, Enrique; Aguillo, Isidro F. (2014). Cibermetría. Midiendo el espacio red. Barcelona: El profesional de la información, Editorial UOC, 190 pp. ISBN: 9788490642337

\section{La web social como nuevo medio de comunicación y evaluación científica de Amalia Mas-Bleda e Isidro F. Aguillo}

Este libro quiere ser, para los académicos, una guía para difundir mejor sus productos; para los profesionales de la información y quienes trabajan en evaluación científica, un catálogo de altmetría y una ayuda sobre nuevas fuentes y métricas; y para el público en general, un lugar donde encontrar nuevos canales de acceso al conocimiento científico.

Mas-Bleda, Amalia; Aguillo, Isidro F. (2014). La web social como nuevo medio de comunicación y evaluación científica. Barcelona: El profesional de la información, Editorial UOC, 208 pp. ISBN: 9788490649220
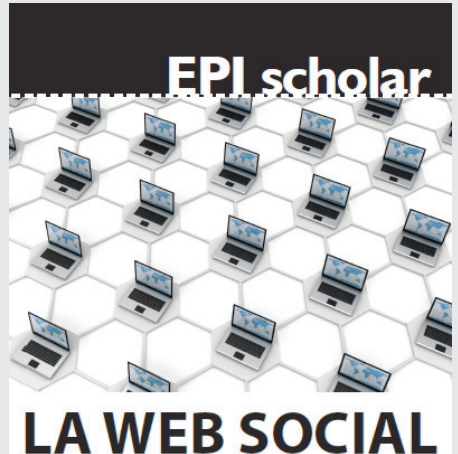
COMO NUEVO MEDIO DE COMUNICACIÓN Y EVALUACIÓN CIENTÍFICA AWAALA MAS-BLEDA ISIDRO F. AGUILLO

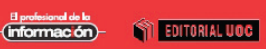

